\title{
Counter electrojet features in the Brazilian sector: simultaneous observation by radar, digital sounder and magnetometers
}

\author{
C. M. Denardini ${ }^{1}$, M. A. Abdu ${ }^{1}$, H. C. Aveiro ${ }^{1}$, L. C. A. Resende ${ }^{1}$, P. D. S. C. Almeida ${ }^{1}$, Ê. P. A. Olívio ${ }^{1}$, \\ J. H. A. Sobral ${ }^{1}$, and C. M. Wrasse ${ }^{2}$ \\ ${ }^{1}$ Instituto Nacional de Pesquisas Espaciais, S. J. Campos, SP, Brazil \\ ${ }^{2}$ IP\&D, Universidade do Vale do Paraíba, S. J. Campos, SP, Brazil
}

Received: 30 September 2008 - Revised: 11 February 2009 - Accepted: 20 February 2009 - Published: 3 April 2009

\begin{abstract}
In the present work we show new results regarding equatorial counter electrojet (CEJ) events in the Brazilian sector, based on the RESCO radar, two set of fluxgate magnetometer systems and a digital sounder. RESCO radar is a $50 \mathrm{MHz}$ backscatter coherent radar installed in 1998 at São Luís (SLZ, $2.33^{\circ} \mathrm{S}, 44.60^{\circ} \mathrm{W}$ ), an equatorial site. The Digital sounder routinely monitors the electron density profile at the radar site. The magnetometer systems are fluxgate-type installed at SLZ and Eusébio (EUS, 03.89 $\mathrm{S}, 38.44^{\circ} \mathrm{W}$ ). From the difference between the horizontal component of magnetic field at SLZ station and the same component at EUS (EEJ ground strength) several cases of westward morning electrojet and its normal inversion to the eastward equatorial electrojet (EEJ) have been observed. Also, the EEJ ground strength has shown some cases of CEJ events, which been detected with the RESCO radar too. Detection of these events were investigated with respect to their time and height of occurrence, correlation with sporadic E (Es) layers at the same time, and their spectral characteristics as well as the radar echo power intensity.
\end{abstract}

Keywords. Ionosphere (Electric fields and currents; Ionosphere-atmosphere interactions) - Radio science (Ionospheric physics)

\section{Introduction}

It is well established that the two types of irregularities identified in the equatorial electrojet are generated by a plasma instability, which has two driving terms (Fejer and Kelley, 1980). One term involves ion inertia and is referred to as the two-stream term (type I irregularities). The other term

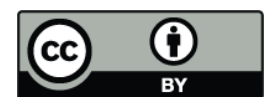

Correspondence to: C. M. Denardini (denardin@dae.inpe.br) involves plasma density gradient and is known as the gradient drift term (type II irregularities). However, both of them depend on the E-Region electric field. From HF and VHF backscatter radar studies of the daytime equatorial electrojet (Fejer and Kelley, 1980, and references therein) we know that the phase velocities of the field aligned plasma irregularities generated by type II mechanism present a normal westward drift in the presence of an upward polarization electric field and a positive electron density gradient. Therefore, inversion in the polarization electric field and in the electron density gradient may cause inversion in the drifts of such irregularities. For type I irregularities, electric field inversion from strong upward to strong downward shall be enough to reversal of the drift velocity to eastward. Obviously, drift inversions related to the polarization electric field will be followed by inversion in the EEJ current as well, which can be detected by its signature in horizontal component of the Earth magnetic field $(H)$ of the magnetic field measured by ground magnetometer close to dip equator.

From a detailed simulation study, Hanuise et al. (1983) explained the possible reversal of the east-west electric field during the above CEJ event as due to an abnormal combination of global scale tidal wind modes, i.e., strong semidiurnal $(2,2)$ and $(2,4)$ modes and a weak diurnal $(1,-2)$ mode and assuming that the contribution of the diurnal tide to the altitude-integrated current flow cancels out. Recently, a study on the CEJ was published (Gurubaran, 2002) in which the author applied the method of natural orthogonal components to the ground geomagnetic data in order to separate the normal and the abnormal field variations (Vertlib and Wagner, 1970; Faynberg, 1975). They showed evidences that there is an additional current system with westward flow in the afternoon hours detected by the ground stations at electrojet latitudes and northward flow at noon hours detected at low and mid latitudes, i.e., there is an additional current system that is superposed to the normal Sq current vortex. They also affirmed that it is mainly due to high order components

Published by Copernicus Publications on behalf of the European Geosciences Union. 
of ground geomagnetic data and is related to the CEJ. Tarpley (1973) stated that the position of the Sq focus would alter the strength of the equatorial electrojet. Kane (1976) showed that the daily range in the $H$ field at electrojet stations is poorly correlated with the range at low latitudes, but the correlation improves when the displacement of the Sq focus is taken into account.

So far, we have mostly mentioned the works of authors who believe that an appropriate combination of tidal modes, like the $(1,-2),(2,2)$ and $(2,4)$ modes, would generate the reverse current at the magnetic equator which causes the negative perturbation in the ground magnetic field variations. Nevertheless, other authors believe that there could be local causative events responsible for reversing the EEJ current into CEJ instead of global events. Most of them believe that vertical winds and gravity wave associated shearing winds are capable of producing such current reversals in narrow latitude and altitude regions (Raghavarao and Anandarao, 1987; and references therein). Their contention is supported by frequent manifestations of CEJ in a narrow latitudinal zone and often in a narrow longitude sector.

According to Somayajulu and Viswanathan (1987) many events of counter electrojet have been observed in the Indian sector. It was also found that even weak counter electrojet events could be observed with the backscatter radar if a negative electron density gradient is present during such events, which occur in the presence of blanketing Es layers. They also observed strong local shears in the E-region wind structures, which they stated to be responsible for some CEJ events. Somayajulu et al. (1994) presented Doppler spectra observed with coherent backscatter radar during a CEJ event. The authors observed type II spectra with CEJ event intensity at about $-10 \mathrm{nT}$ in the presence of blanketing Es layers and type I spectra with CEJ event intensity between -35 and $-40 \mathrm{nT}$ in the absence of blanketing Es layers. The altitude of the echoing region was $95-100 \mathrm{~km}$ (in the presence of blanketing Es layers) during the observations of type II spectra and ranged from 100 to $108 \mathrm{~km}$ when type I waves are observed. Using a HF radar at Addis-Ababa (Eastern African sector), Crochet et al. (1979) observed two stream waves having phase velocity greater than the ion-acoustic velocity in the presence of normal upward (stabilizing) gradient during the CEJ event of 21 January 1977. Recently, Woodman and Chau (2002) reported the first VHF radar observations of two stream waves during CEJ events since the first 40 years of existence of the observatory at Jicamarca (Peruvian sector). They stated that at the beginning and ending of the CEJ events they found what they called "the closest pure two-stream linear instability conditions". Along with the radar observations they showed that these CEJ events reached $-200 \mathrm{nT}$.

In between these above mentioned studies there is the study from Alex and Mukherjee (2001), which compared CEJ events at the two equatorial stations Trivandrum and Addis Ababa ( $40^{\circ}$ apart in longitude). They used Harmonic
Analysis on the $H$ component of the Earth magnetic field for the days chosen for the period between 1991 and 1993. Their main conclusion is that there are some differences in CEJ events when observed in different longitudinal sectors. But the most important aspect is that they attributed the observed differences to local wind shears that modify jet fields (especially during the noon hours) in addition to the changes in the large scale global wind dynamo, i.e., they stated that there is a combination of local and global effects that roles the development of CEJ events.

Despite several studies being done during the last decades, the mechanism that drives the CEJ current under quiet geomagnetic conditions is still an open question. The only agreement is that the counter electrojet is more frequently observed during solar minimum solstice months (Mayaud, 1977). Also, all the above-mentioned studies have been made in the Indian, the Eastern African and the Peruvian sectors mainly based on magnetometers. Thus, the present work covers a different longitudinal zone by examining some CEJ features based on ground geomagnetic data, on digital sounder data and on VHF coherent radar data in the Brazilian sector, over which a large magnetic declination angle (being $\sim 20^{\circ} \mathrm{W}$ ) exists. For this initial analysis, we have chosen data from 2002 due to the VHF radar observations availability. However, 2002 was close to the solar maximum and only few CEJ cases were detected during the radar soundings, in agreement with Mayaud (1977). We will examine cases of evening, afternoon and morning CEJ events.

\section{Data analysis}

In order to determine the strength of the magnetic effect of the EEJ current at the ground level (named EEJ ground strength for simplification), the difference between the $H$ component variations of the Earth magnetic field ( $\Delta H$ variation) at São Luís - SLZ $\left(2.3^{\circ} \mathrm{S}, 44.2^{\circ} \mathrm{W}\right.$, dip: -0.5$)$ and Eusébio - EUS $\left(3.89^{\circ} \mathrm{S}, 38.44^{\circ} \mathrm{W}\right.$, dip: -12.5$)$ stations $\left(\Delta H=\Delta H_{\mathrm{SLZ}}-\Delta H_{\mathrm{EUS}}\right)$ was evaluated. Such evaluation was made for all days in 2002, except for most of the days in June and the first two weeks in July due to a lack of data from EUS. The basic treatment of the magnetic data at each station is to eliminate outlier values from the measured components, based on a 3 rd order polynomial fitting. Thereafter, the five quietest days in a month are chosen and their local midnight values averaged. Then, the station $H$ component variations are normalized to the difference between the $H$ component values and the mean midnight values for the five quietest days. Finally, variation of the EEJ ground strength is estimated by taking the difference between the $\Delta H$ values at a station at the dip equator and that at a station nearby the dip equator (outside the EEJ influence). Concerning about Earth induction effects on magnetic data, some authors claim we should include a factor of $3 / 2$ when calculating the magnetic flux density due to a current sheet (Hargreaves, 1992). 
This factor should be enough for location far from induction anomalies, which is the case of both SLZ and EUS. However, we will not include this factor as well as we will not calculate the integrated current of the EEJ. Instead, we chose to keep EEJ ground strength (given in $\mathrm{nT}$ ) as the strength of the magnetic effect at the ground level caused by the EEJ current sheet.

Data from 3-m irregularities, present in the EEJ, were collected using the RESCO $50 \mathrm{MHz}$ radar at SLZ (for detailed descriptions see, for instance, Denardini et al., 2005). The radar operation schedule follows the Geophysical Calendar or some emerging necessity and the operation is done mainly during daytime. In 2002, the radar operated during 120 days in beam switching mode at the rate of one cycle per minute. The radar beam with a width of $7^{\circ}$ in the East-West plane was switched between magnetic east and magnetic west, having an elevation angle of $60^{\circ}$. The relatively narrow beam allows us to deduce the altitude of the scatter directly from the range. At each beam change, RESCO performed 10 soundings at a rate of 512 non-coded pulses per sounding. For the $\mathrm{E}$ region sounding, the pulse width was set to $20 \mu \mathrm{s}$ and the inter-pulse-period was set to $1 \mathrm{~ms}$. The time delay is set to $600 \mu \mathrm{s}$. After this delay, 16 subsequent samples $20 \mu \mathrm{s}-$ wide ( $3 \mathrm{~km}$ in range, $\sim 2.6 \mathrm{~km}$ in height) are taken in order to cover the height range from about 80 to $120 \mathrm{~km}$. For each day of radar sounding, we obtained two Range-TimeIntensity (RTI) maps (one per beam position) and 32 spectrograms (16 per beam position). Each spectrogram, consisted of $\sim 300$ spectra, and the RTI maps were incoherently integrated to result in a time resolution of $2 \mathrm{~min}$. The aliasing frequency of the spectrograms is $250 \mathrm{~Hz}(\sim 750 \mathrm{~m} / \mathrm{s}$ for the RESCO radar) with $\sim 1 \mathrm{~Hz}(\sim 3 \mathrm{~m} / \mathrm{s})$ of frequency resolution. From the spectrograms, we estimate the vertical drift profile of the electrojet irregularities, which is an indicative of the local electric fields and, in turn, of the EEJ current. In the present analysis, we used the westward beam only. Therefore, we limited the study to one RTI map per day with the 16 corresponding spectrograms.

Ionospheric profiles are ordinary electron density profiles obtained from the digital sounder installed in SLZ (for detailed descriptions see, for instance, Reinisch et al., 1989). The digital sounder operates continuously during all days of the years and electron density profiles are taken every $15 \mathrm{~min}$ with frequencies in the range from 0.5 to $15.0 \mathrm{MHz}$, with $0.5 \mathrm{MHz}$ of frequency step. From them it is possible to identify sporadic E layers (Es) and to examine the critical frequency of the Es layer ( $f o E s)$ and the cut-off frequency of the Es layer ( $f b$ Es).

\section{Results and discussions}

\subsection{EEJ ground strength from magnetometer systems}

The diurnal variations of the EEJ ground strength; and diurnal variations of the $H$ component of the Earth magnetic field measured at São Luís $\left(\Delta H_{\mathrm{SLZ}}\right)$ and Eusébio $\left(\Delta H_{\mathrm{EUS}}\right)$ in 2002 are shown in the Fig. 1 . Both $\Delta H_{\text {SLZ }}$ and $\Delta H_{\text {EUS }}$ show a clear diurnal variation characterized by an increase during the morning period until around local midday and a decrease in the afternoon. Such variations are typical variations attributed to the E-region solar quiet (Sq) system (Heelis, 2004). $\Delta H_{\text {SLZ }}$ variation peaks at about $180-200 \mathrm{nT}$ during the D months (January, February, November and December) and peaks at about 100-150 nT during the $\mathrm{J}$ months (May, June, July and August). $\Delta H_{\text {EUS }}$ variation peak values varies around $100 \mathrm{nT}$, and are also observed to be stronger during the D months. Since these differences in the variations of the $H$ component is observed at the two stations, it should consequently be associated with the $\mathrm{Sq}$ system, and therefore affect the EEJ ground strength. Indeed, the peak values of the EEJ ground strength also show a seasonal variation being around $50 \mathrm{nT}$ during $\mathrm{J}$ months and around $100 \mathrm{nT}$ during D months. However, such peak strength variations are not clearly observed in the radar data on the plasma irregularities, which in turn reflect the E-Region electric fields. Both $\Delta H_{\mathrm{SLZ}}$ and $\Delta H_{\mathrm{EUS}}$ also show a modulation in the baseline value, which is especially clear during some months at EUS. Take, for example, the $\Delta H_{\text {EUS }}$ between 26 March and 18 April or between 27 July and 20 August. These $\sim 27$-day oscillations are related to the solar rotation and should not reflect directly upon the calculation of the EEJ ground strength (H. McCreadie, private communication, 2005) since we take the difference between the two stations close apart. Also, a latitudinal correction on the $H$ variation could be applied before taking the difference between the components of the two stations. This would make the calculation of the magnetic flux density due to the EEJ current sheet more accurate. However, we did not correct it because we used the variation of the $H$ component relative to the midnight value $(\Delta H)$ and because the stations are very close apart so that the correction can be neglected. Also, we did not intend to calculate the integrated current of the EEJ. Therefore, the EEJ ground strength should be seen as an indicative of the EEJ current intensity variation but not the EEJ current itself.

Even though, the EEJ ground strength shows remarkable characteristics of the Sq influence on the EEJ behaviour. The diurnal variations of the EEJ strength at the ground reveal a heart beating like behaviour. There is a relatively short period of low negative amplitude excursion of EEJ strength before the dawn followed by a positive amplitude excursion during daytime. The negative amplitude excursion is a manifestation of westward morning electrojet. The dawn occurs earlier at the E-region heights then at the ground level. This means the photoionization process (which increases substantially 

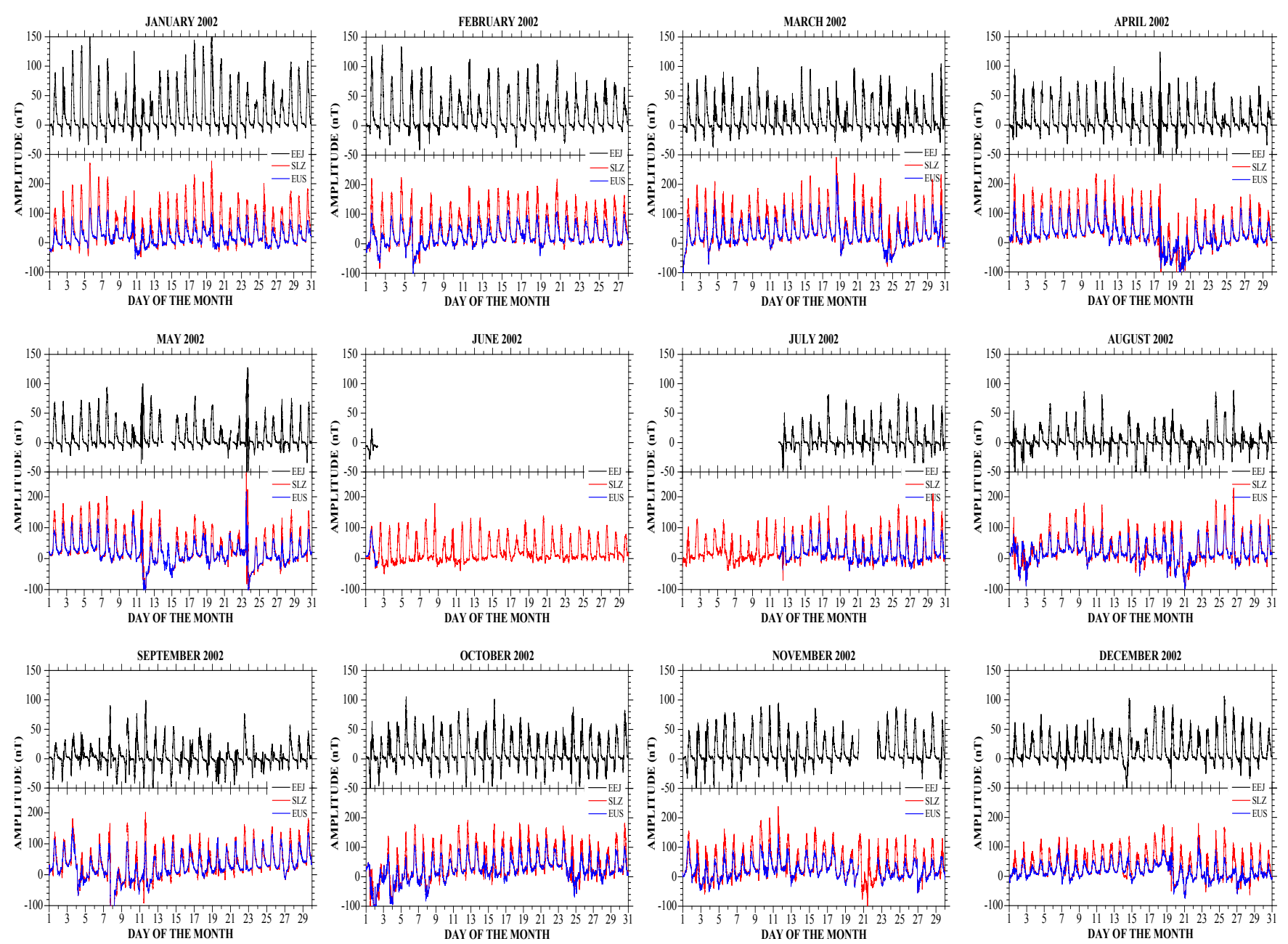

Fig. 1. Diurnal variations of (top panel) the EEJ ground strength and (bottom panel) diurnal variations of the $H$ component of the Earth magnetic field measured at (red) São Luís and (blue) Eusébio in 2002.

the local conductivities) have already started at EEJ heights when it is to dawn at the ground, but, more important, it also means that the zonal electric field did not invert yet. Since westward currents shall induce southward magnetic fields below them, which contribute to reduce the $\mathrm{H}$ component of the Earth magnetic field at the ground level, this simple method can be used to infer the time of inversion of the EEJ electric field.

Some minutes after dawn, the zonal electric field inversion is completed and the vertical polarization electric field starts to drive the EEJ current to its diurnal direction. It is due to the higher conductivities during daytime that the EEJ ground strength reaches positive amplitudes higher than the negative ones. This is observed even during clearly disturbed periods like 24-25 March, 18-22 April, 23-25 May, 30 September8 October, and 19-23 December. What seems to vary is the amplitude of the negative excursion, the positive excursion or both of them, depending upon the intensity of the magnetic disturbances.

\subsection{CEJ event occurrences}

A typical behaviour of the EEJ in the Brazilian sector (as seen in RTI maps) is characterized by the back-scattering region of 3-m plasma irregularities starting to be observed at around 08:00-08:30 LT every day (see for example, Denardini et al., 2005). Such irregularities appear even when the EEJ current is still weak (less than $10 \mathrm{nT}$ ) to show a clear signature in the EEJ ground strength. After some variability during the early morning hours, which is still a matter of study, there is a tendency of this backscattering region to stabilize at around $103 \mathrm{~km}$ of altitude. After around 14:00 LT the whole scattering region starts to rise as shown in the previous work by Denardini et al. (2005), when a tentative explanation given in terms of the chemistry of the E-Region partially justified the observed rising. Backscattered echoes strength becomes strongly reduced after about 18:00 LT and they normally vanish after about 19:00 LT, except for some cases reported by Denardini et al. (2006). A typical behaviour of the EEJ 

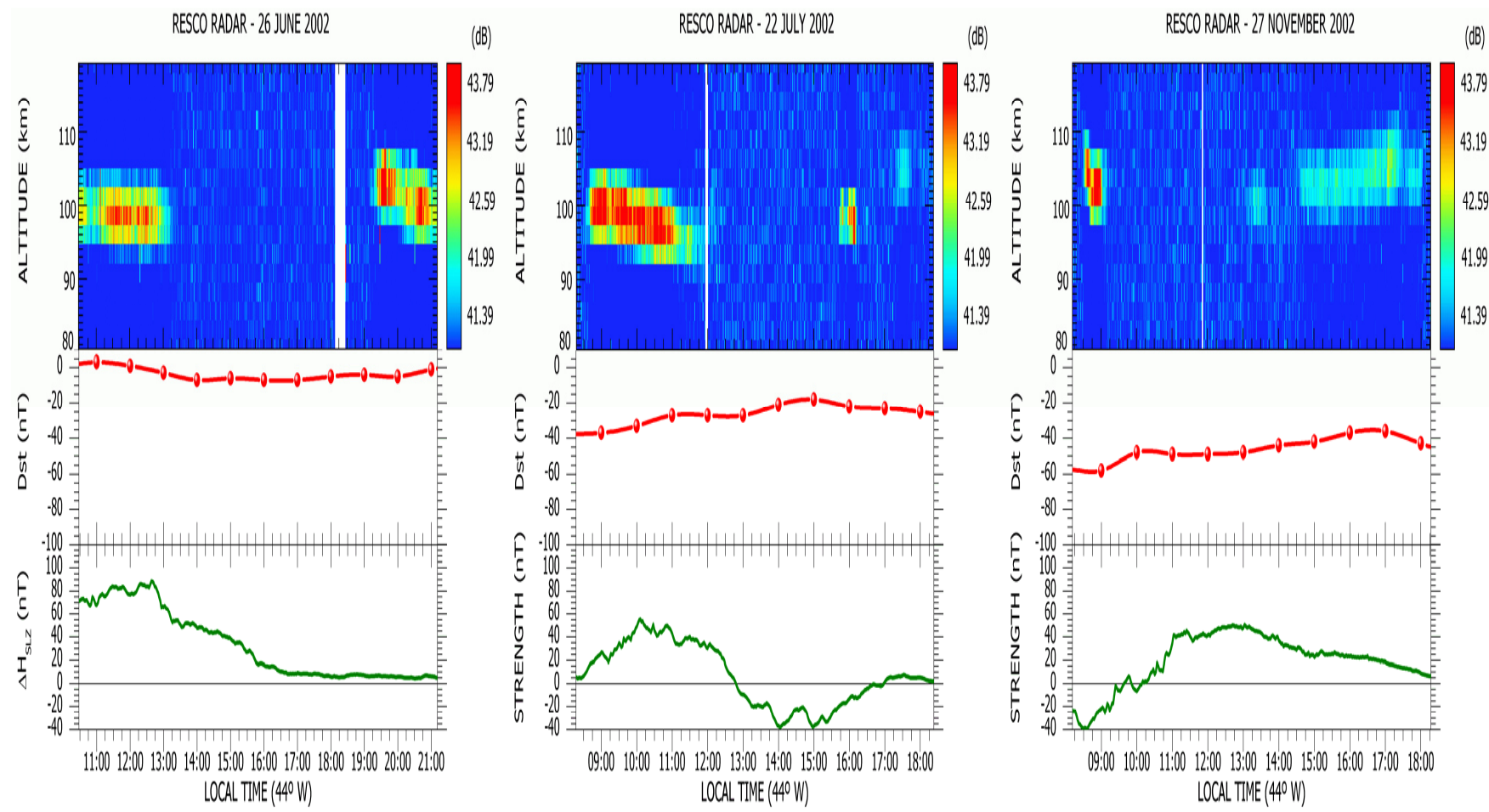

Fig. 2. Range Time Intensity (RTI) maps obtained from the RESCO radar on (from the left) 26 June 2002, 22 July 2002 and 27 November 2002, when an evening, an afternoon and a morning CEJ events have been detected, respectively. Corresponding $D_{s t}$ index is on the middle panel and the variation of EEJ ground strength $\left(\Delta H=\Delta H_{\mathrm{SLZ}}-\Delta H_{\mathrm{EUS}}\right)$ obtained from magnetometers on the bottom panel. Note that on 26 June the EEJ ground strength was replaced by the $\Delta H_{\mathrm{SLZ}}$ variation.

ground strength follows a basic pattern. It shows a slowly intensification in the morning hours until reaches a quiescent value around midday. It remains about this value with some small-scale fluctuations, typically less then $2 \mathrm{nT}$ (related to a 30 min running average) in a time scale no longer then $15 \mathrm{~min}$. After about 16:00 LT it slowly decreases to zero.

CEJ events usually occur when such a pattern described above is substantially altered and very few cases were observed during 2002. The clearest events were observed during the solstice months in agreement with previous work (Mayaud, 1977), but during a solar maximum for the present case. Figure 2 shows RTI maps in which an evening, an afternoon and a morning CEJ event were detected, respectively. The corresponding EEJ ground strengths detached from the Fig. 1 were plotted in the bottom panel of each graph except on 26 June, when the EEJ ground strength was replaced by the $\Delta H_{\text {SLZ }}$ variation since we have a lack of data from EUS on many days in June and July. The $D_{s t}$ index variation were also plotted to characterize the corresponding magnetic disturbance level. It should be noticed that the time scales of the maps are different from each other, due to day-to-day changes in the radar operation schedule. Moreover, it is important to mention that one given CEJ event cannot be identified from the RTI map alone as being a CEJ or a normal morning EEJ. The identification is made with the help of the corresponding spectrograms that are presented in Fig. 3.

\subsubsection{The evening CEJ event}

On 26 June, the radar operation started at 10:30 LT. At that time, the RTI map reveals that EEJ irregularities seemed to be weakly developed as seen by the backscattered echo power strength. In addition, the $\Delta H_{\mathrm{SLZ}}$ variation reveals a weak induced current since normal $H$ component peak values range from 100 to $200 \mathrm{nT}$, as mentioned previously. The spectrograms obtained on June 26 show the irregularities observed until around midday were drifting westward (negative Doppler velocities when sounded by a westward radar beam). After about 13:15 LT, the irregularities responsible for scattering back the radar signal disappeared. It was simultaneously followed by a gradual reduction in the $\Delta H_{S L Z}$. Abdu et al. (2006) showed that during storm time the Sq system can be affected by wind circulations due to the disturbance dynamo and/or by auroral electric field penetration. The $D_{s t}$ index did not show any evidence of disturbance, however. Digisonde data (not shown here) show no electron density gradient inversion or reduction at the E-Region heights by the time of this disappearance. Therefore, we can assume that the electric field, which drives the plasma instabilities, has been reduced to a value lower than necessary to trigger them on. Also, no significant reduction in the local conductivity should have occurred since the density profile has not been substantially altered. Then, a reduction in the 

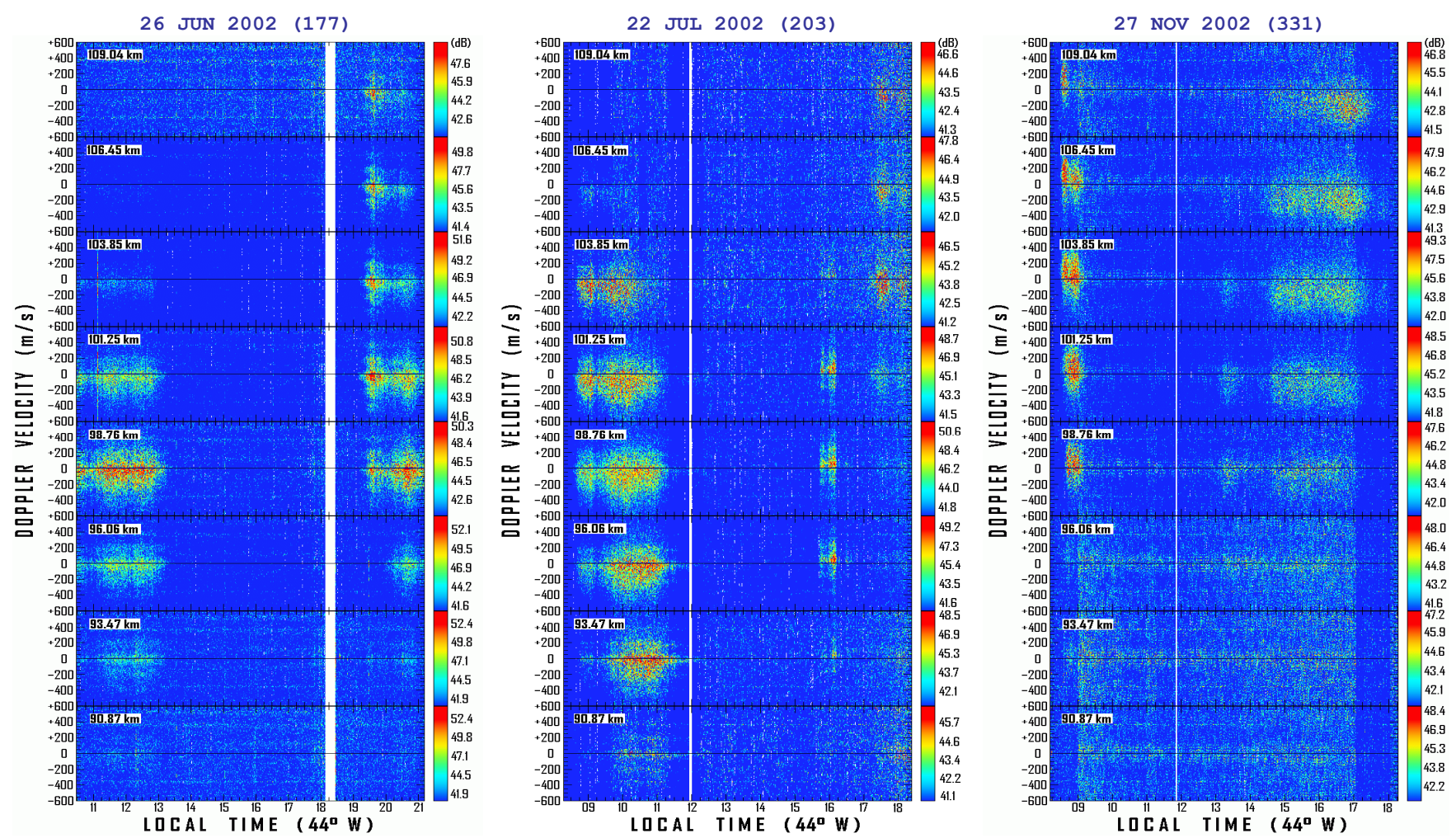

Fig. 3. Set of eight spectrograms covering the range height from 90.9 to $109.0 \mathrm{~km}$, obtained on (from the left) $26 \mathrm{June} 2002,22 \mathrm{July} 2002$ and 27 November 2002, when an evening, an afternoon and a morning CEJ events have been detected, respectively. The central height of each spectrogram, which covers $2.6 \mathrm{~km}$ of altitude, is informed on the top left corner of the corresponding spectrogram and the power scale on the right side is given in $\mathrm{dB}$ units.

induced current strength indicates a reduction in the electric field, which is most likely due to neutral atmosphere effects rather than disturbance dynamo effects as judged by the low disturbance level confirmed by the $D_{s t}$ index.

Later on 26 June, after about 19:00 LT when irregularities are likely to drift eastward at different range heights (Fejer et al., 1975), three consecutive evening CEJs are observed. The first event occurred at 19:15 LT and lasted for $\sim 30 \mathrm{~min}$. The CEJ was located between 98.8 and $109 \mathrm{~km}$ and showed westward velocities lower than $100 \mathrm{~m} / \mathrm{s}$. The second event started just after 20:15 LT and lasted for $15 \mathrm{~min}$. The CEJ was located in a very narrow range height, between 98.8 and $101 \mathrm{~km}$. Westward velocities lower than $100 \mathrm{~m} / \mathrm{s}$ were also detected. The third event started at 20:30 LT and lasted for 30 min like the first one. However, the CEJ was located between 93.5 and $106.5 \mathrm{~km}$. The observed velocities also diverged from the first event. Westward velocities close to $200 \mathrm{~m} / \mathrm{s}$ were detected at $98.8 \mathrm{~km}$ height. Velocities lower than $360 \mathrm{~m} / \mathrm{s}$ are characteristics of type II instabilities, which are generated by the gradient-drift mechanism. As mentioned before, this mechanism requires the density gradient to be parallel to the electric field.

The density gradient sharp enough for irregularities development is a feature of the bottom side E-Region density profile during daytime or can be achieved in the presence of an Es layer at the proper height anytime. Figure 4 shows a sequence of ionograms acquired on 26 June (left column) covering the height range from 50 to $500 \mathrm{~km}$, between 19:00 and 21:45 LT where it is possible to identify the presence of blanketing Es layers during these CEJs with type II irregularities, indicating that these radar echoes are due to irregularities at the sporadic E layer like Somayajulu and Viswanathan (1987) reported. In the present case, all the Es are equatorial type of Es traces (type q), which are commonly found during day time in the vicinity of the magnetic dip equator. The $f b \mathrm{Es}$ did not exceed $4 \mathrm{MHz}$ for the first CEJ, but reached $5 \mathrm{MHz}$ during the second and the third events. The virtual height of the Es layer peaked at about $110 \mathrm{~km}$ during the two first events and rose to around $150 \mathrm{~km}$ after 20:30 LT. Therefore, the density gradients for all the three CEJ events observed on 26 June are upward.

The electric field is expected to be mainly downward after sunset under quiet time conditions. However, based on the direction of irregularity displacement (westward) and on the echoes height shown in both the RTI map and the ionogram, it has to be upward. Otherwise, these echoes would not be from plasma irregularities caused by gradient-drift instabilities or the theories do not fit. The explanation for such 

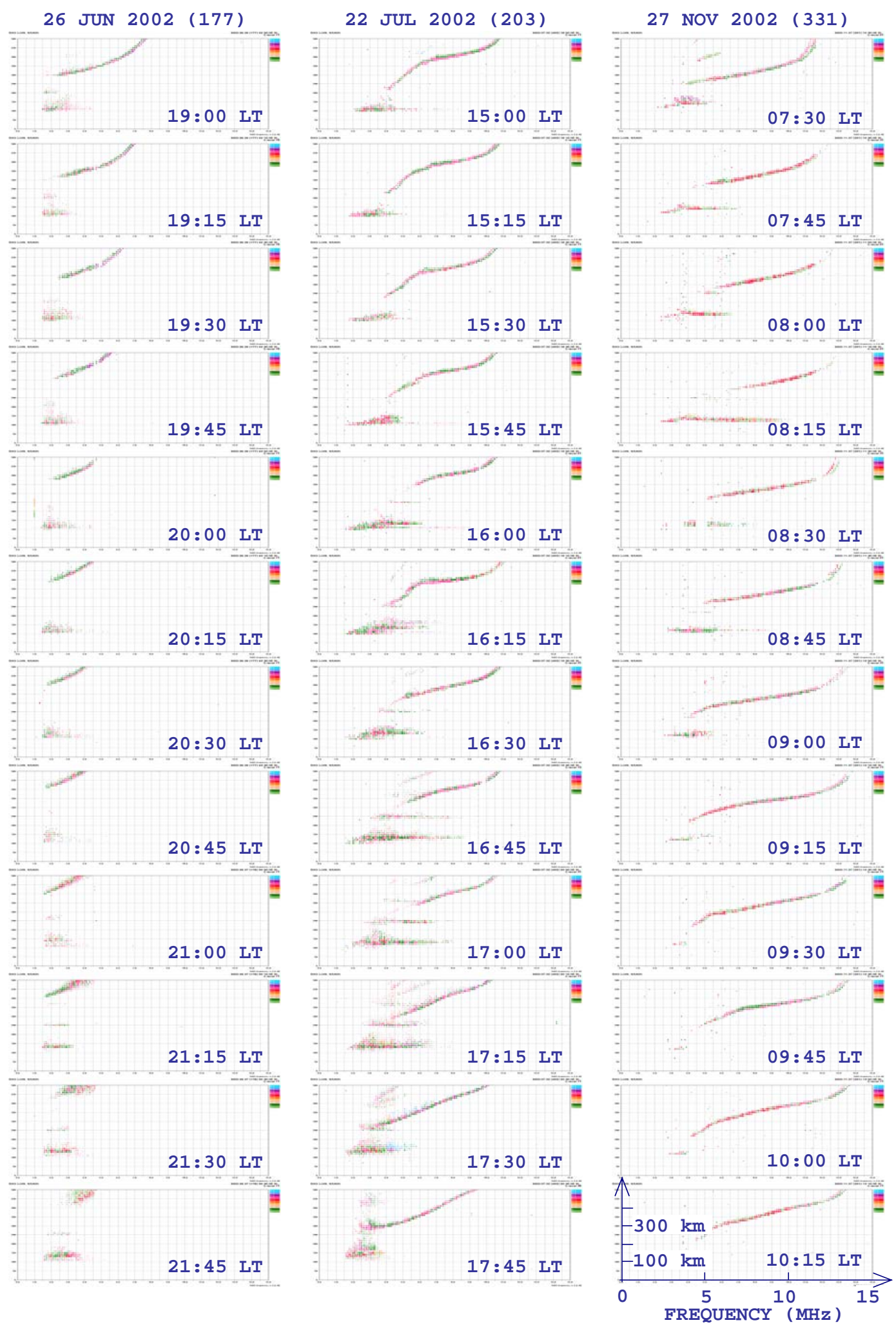

Fig. 4. Sequence of ionograms covering the height range from 50 to $500 \mathrm{~km}$, obtained on 26 June 2002 (left), on 22 July 2002 (middle) and on 27 November 2002 (right). 
upward electric field is still an open question. Some authors use to attribute it to neutral wind shears that drags the plasma to cause a localized polarization electric field.

Since we have no data from the neutral atmosphere, we have performed a wavelet analysis of the $\Delta H_{\text {SLZ }}$ (as an indicative of the EEJ electric field) using the continuous wavelet transform in order to search for possible wave-type oscillations that could lead to an indication of wind shear. Wavelet transform was chosen due to its ability to locate in time the oscillations, which allow us to check if their time of occurrence matches with the time of occurrence of the CEJ. Indeed, the continuous wavelet transform (CWT) of a discrete sequence of samples $\left(x_{n}\right)$ is defined as the convolution of the samples with a scaled and translated version of the wavelet-mother. Alternatively, it can be treated as the inverse Fourier transform of the product in the frequency domain as proposed by Torrence and Compo (1998):

$$
W_{n}(s)=(2 \pi s / \delta t) \sum_{k=0}^{N-1} \hat{x}_{k} \hat{\psi}_{0}^{*}\left(s \omega_{k}\right) \exp \left[i \omega_{k} n \delta t\right],
$$

where $s$ is the wavelet scale, $k$ is the frequency index, $\delta t$ is the time resolution, $N$ is the total number of points, $\hat{x}$ is the Fourier transform of the time series, the angular frequency $\left(\omega_{k}\right)$ is defined as $+2 \pi k / N \delta t$ when $k \leq N / 2$ and $-2 \pi k / N \delta t$ when $k>N / 2$, and $\hat{\psi}_{0}^{*}\left(s \omega_{k}\right)$ is the complex conjugate of the wavelet-mother. We used Morlet wavelet-mother, which is a plane wave modulated by a Gaussian envelope of unit width (Farge, 1992) given by:

$\hat{\psi}_{0}\left(s \omega_{k}\right)=\pi^{-1 / 4} H(\omega) \exp \left[-\left(s \omega-\omega_{0}\right)^{2} / 2\right]$

because it is the appropriated type for searching periodicities on the data sets. The Heaviside step function $H(\omega)$ is equal to zero at for negative frequencies $(\omega)$ and equal to one otherwise. Finally, the space-scale energy density for a time series is defined as

$E_{n}(s)=\left|W_{n}(s)\right|^{2} / s \quad$.

On the upper panel of Fig. 5 we show the Space-Scale Energy Density Map (bottom left graph) with the corresponding global wavelet spectrum (bottom right graph) and its spacescale energy density colour scale (upper right graph), obtained from wavelet analysis of the $\Delta H_{\mathrm{SLZ}}$ variations (upper left graph) between 11:00 and 21:00 LT on 26 June 2002 . The cone-of-influence is superimposed on the very bottom part of the map. However, this map does not help solving the origin of the upward electric field that was observed on 26 June. Due to the low values of $\Delta H_{\mathrm{SLZ}}$ in the evening, the small scale fluctuations show no significant energy to be detected in the analysis. Nevertheless, it will be useful for the other cases.

\subsubsection{The afternoon CEJ event}

On 22 July, the RESCO radar started to operate at 08:15 LT and stopped sounding at about 18:30LT. The RTI map for this day (middle panel of Fig. 2) reveals that the development of EEJ plasma irregularities occurred in a way similar to that on 26 June. They started to be observed in the morning hours and lasted until close to midday. However, the echo power intensity was stronger in the present case than that on 26 June. Another difference is the descent of the scattering region after $\sim 09: 45 \mathrm{LT}$. The centre of the EEJ scattering region (EJC) descent from $99.9 \mathrm{~km}$ (09:45 LT) to $94.4 \mathrm{~km}(11: 15 \mathrm{LT})$. Then, similarly to what have happen on 26 June, the plasma irregularities responsible for scattering back the radar radio signal vanished at the moment the EEJ ground strength reduced below $20 \mathrm{nT}$. Nevertheless, contrary to the previous case, the $D_{s t}$ index did show evidence of moderate magnetic disturbance throughout the day, ranging between -40 and $-20 \mathrm{nT}$. Also, the EEJ ground strength variation showed an inversion of the EEJ current at 13:00 LT that lasted until about 17:00 LT. During the negative excursion, the EEJ ground strength reached minima of $-40 \mathrm{nT}$ at 14:00 and 15:00 LT. In addition, at about 15:45 LT a scattering region was observed by the RESCO radar with its centre height of at $99.2 \mathrm{~km}$ of altitude. It lasted until 16:15 LT when the EEJ ground strength rose up to $-18 \mathrm{nT}$. The spectrograms in the middle panel of Fig. 3 confirm the descent of the scattering region since the height distribution of the echo power changed from about $96-103 \mathrm{~km}(\sim 09: 00 \mathrm{LT})$ to about 93-101 km ( 11:00 LT) and the centre height of CEJ event is narrowed in both altitudinal and temporal dimensions. It also reveals very small type II plasma irregularities positive Doppler velocities, which is the opposite to what is expected for a normal EEJ observed with the westward RESCO radar beam, but is in agreement on what is expected for a CEJ event. Later in the evening the normal EEJ conditions seems to be re-established and a weak scattering is observed between 101 and $109 \mathrm{~km}$ of altitude.

The evidence of moderate magnetic disturbance shown by the $D_{s t}$ index indicates that the disappearance of the irregularities around midday could be due to neutral wind circulations changes caused by the disturbance dynamo effect on the Sq system as stated by Hanuise et al. (1983) and Abdu et al. (2006). Regarding the scattering region during CEJ event, the reversal into the Doppler velocities with respect to the normal condition indicates that both the polarizations electric field and the density gradient are downward. A downward density gradient can be achieved due to the presence of sporadic E-layers at lower altitudes, which is the present case as revelled by the set of ionograms shown in the Fig. 4 (middle panels). The time scale $(30 \mathrm{~min})$ of the CEJ event suggests that the downward electric field that drove the irregularities can be caused by prompt penetration (PP) of auroral electric fields. An analysis of the Auroral Index (AE) evolution on 22 July, shows no clear evidence of auroral electric fields that match with the time of occurrence of the CEJ event, however. The closest AE index intensification occurs one hour before the CEJ event. Therefore, an abnormal combination of global scale tidal wind modes (Hanuise et al., 
26 JUN 2002
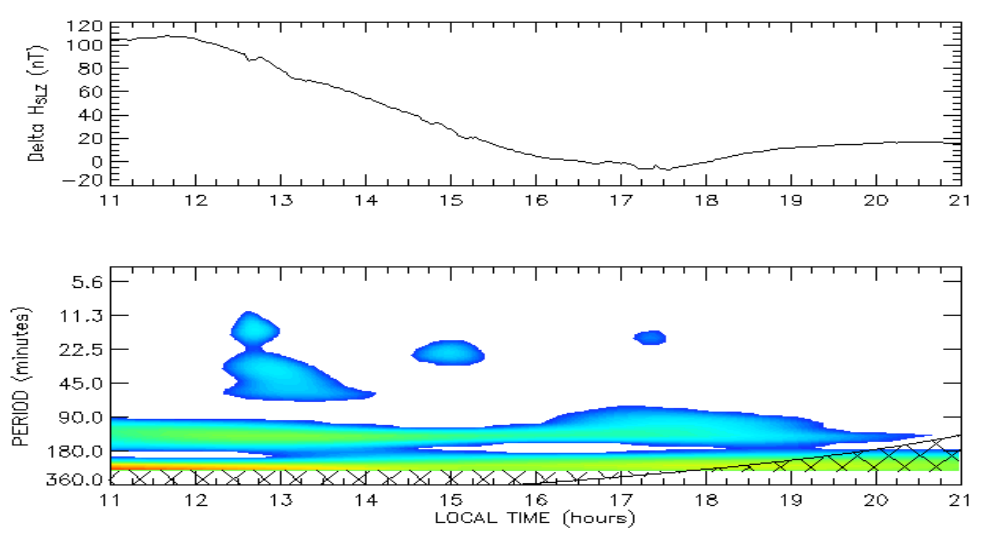

22 JUL 2002
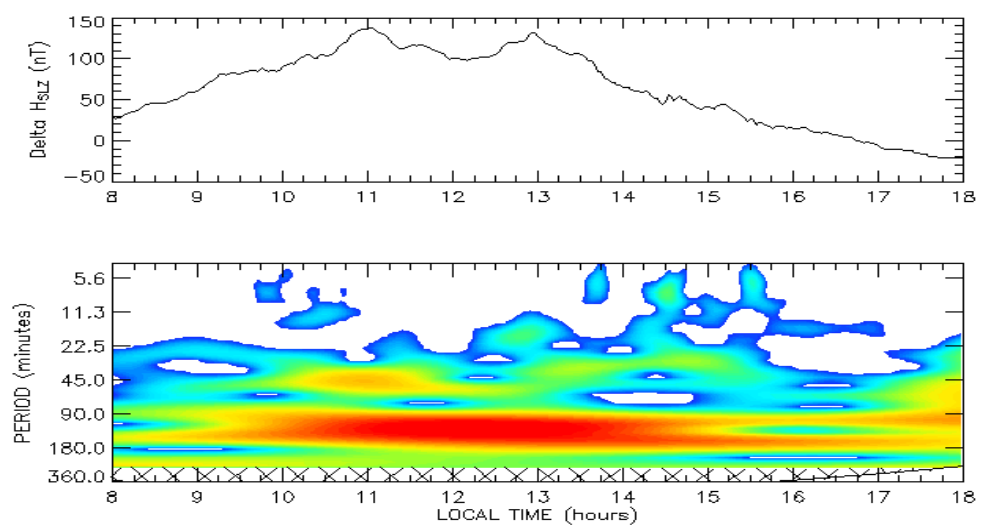

27 NOV 2002
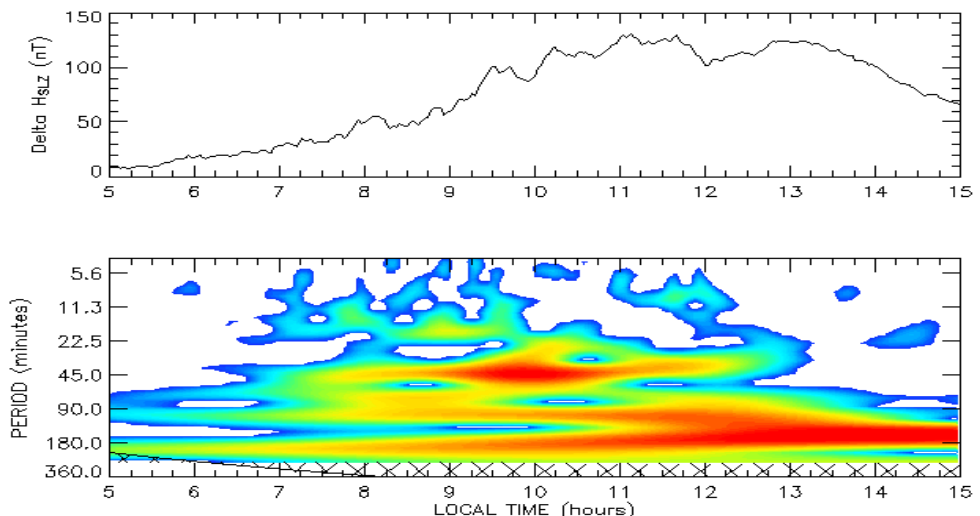
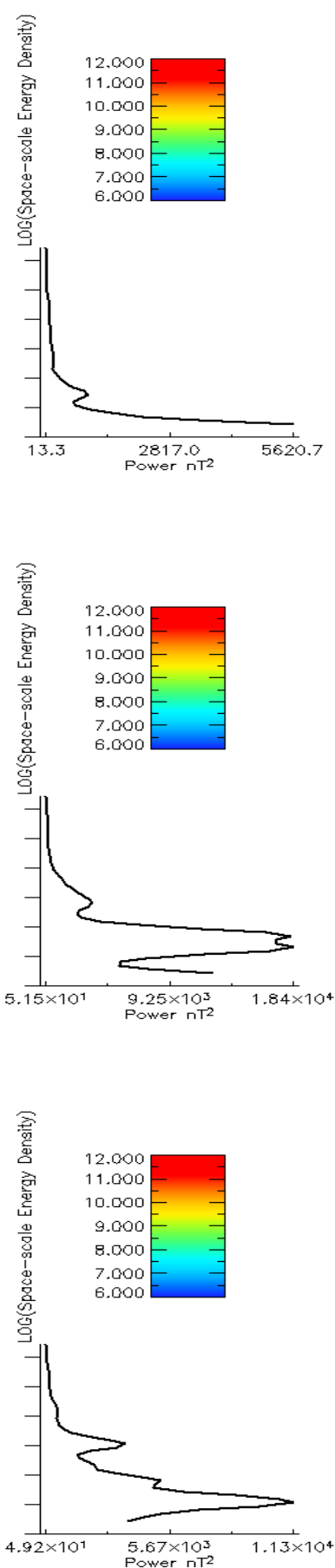

Fig. 5. Space-Scale Energy Density Map (bottom left graph) with the corresponding global wavelet spectrum (bottom right graph) and its space-scale energy density color scale (upper right graph), obtained from wavelet analysis of variations of the $H$ component of the Earth magnetic field measured at São Luís (upper left graph) on 26 June 2002 (upper panel), on 22 July 2002 (middle panel) and on 27 November 2002 (bottom panel).

1983), or vertical winds and gravity wave associated shearing winds (Raghavarao and Anandarao, 1987) are most likely to have caused the electric field inversion. In order to check for wave-like behaviour in the EEJ we have performed the same wavelet analysis of the $\Delta H_{\mathrm{SLZ}}$ between 08:00 and 18:00 LT as we did for 26 June. The analysis results are shown on the 
middle panel of Fig. 5. The Space-Scale Energy Density Map shows a dominant region where the induced current has a wave-like behaviour with two oscillations having 90-min and 180-min periods that mix together after 09:00 LT to become one oscillation with period close to $2 \mathrm{~h}$ and turns back to the two previous oscillations after 14:00 LT. There also is a clear region of a wave-like behaviour with period of the order of $45 \mathrm{~min}$ in the induced current pattern between 10:00 and 12:30 LT. This turned to a wave-like behaviour with period of the order of $30 \mathrm{~min}$ between 12:30 and 15:45 LT. Some others secondary peaks can be observed during all this period. But from 15:45 to 16:30 LT, three clear spectral components are also present having 15-min, 30-min and 60-min periods. Therefore, we assume the electric field inversion to be due to gravity wave associated shearing winds, since the CEJ event was limited between 15:45 and 16:15 LT.

\subsubsection{The morning CEJ event}

On 27 November, the observed CEJ event started at about 08:30 LT and lasted until 09:00 LT, when the EEJ ground strength rose up from -40 to $-20 \mathrm{nT}$. During this event, the irregularities presented positive (eastward) Doppler velocities and were located in the upper part of the scattering region (between 98.8 and $109 \mathrm{~km}$ ). The $D_{s t}$ index (around $-60 \mathrm{nT}$ ) clearly indicates disturbed magnetic conditions throughout the day. This is the only record we have with strong EEJ early in the morning (later then 08:00 LT) showing plasma irregularities that have positive (eastward) Doppler velocities. Nevertheless, to assure this is a CEJ event we also looked at the EEJ ground strength. The latter reveals that the EEJ current reached close to zero before 08:15 LT, was followed by a negative intensification until 08:30 LT and reduced to zero at 09:30 LT. In other words, the slow normal transition from negative to positive EEJ ground strength excursion (before to $08: 15 \mathrm{LT}$ ) seen in most of quiet days was followed by a negative excursion after the inversion, which characterizes a CEJ event. The Doppler velocities ranged from 50 to $200 \mathrm{~m} / \mathrm{s}$, indicating type II irregularities. Then, a downward density gradient and a downward electric field are needed for their development. The set of ionograms shown in the Fig. 4 (right panels) reveal the presence of a strong sporadic E-layer (foEs $\sim 10 \mathrm{MHz}$ ) at about $95-100 \mathrm{~km}$, which also means a strong density gradient and explains the downward density gradient in agreement with Somayajulu et al. (1994). The electric field is certainly downward due to the clear negative current signature at the ground level, but its origin is always difficult to determine. An analysis of the AE Index evolution on 27 November shows disturbances at the auroral electric fields that matched with the temporal scale of the CEJ event but they occurred 5 to $10 \mathrm{~min}$ before the CEJ event. Therefore, we checked for a wave-like behaviour in the EEJ through a wavelet analysis of the $\Delta H_{\mathrm{SLZ}}$ between 05:00 and 15:00 LT on 27 November. The analysis results are shown on the bottom panel of Fig. 5. The Space-Scale Energy Den- sity Map shows a patter similar to the previous case (22 July) with the presence of the two oscillations having 90-min and 180-min periods. However, this time they mix together close to midday only. Also, the three spectral components having 15-min, 30-min and 60-min period were present between 08:30 and 09:00 LT, during the CEJ event. So, neutral atmosphere dynamics seems to play an important hole in driving the EEJ electric field. The longitudinal coverage of such phenomena can not be specified in the present work, however. In order to confirm the combination of global scale tidal wind modes proposed by Hanuise et al. (1983), instead of the vertical winds and gravity wave associated shearing winds proposed by Raghavarao and Anandarao (1987) we need simultaneous observations in different latitudinal sector based on clusters of instruments like those used in the present work. Moreover, checking which, among the three components, is the responsible for driving the EEJ electric field will demand accurate neutral atmosphere wind measurements.

\section{Conclusions}

The typical diurnal variations of the EEJ ground strength derived from $\Delta H_{\mathrm{SLZ}}$ and $\Delta H_{\mathrm{EUS}}$ and attributed to the E-region Sq system are shown to be stronger during the D months when compared with the rest of the year. Very few cases of CEJ events were observed during 2002. The clearest events were observed during the solstice months, in agreement with the previous work by Mayaud (1977) but during a solar maximum in the present case. All the CEJ events observed in Brazil in 2002 are accompanied by blanketing Es layers, in agreement with the previous work by Somayajulu and Viswanathan (1987). The RESCO radar detected type II spectra with the entire CEJ event but no type I was detected, and the CEJ ground strength signature reached $-40 \mathrm{nT}$, which differs from the conditions observed by Somayajulu et al. (1994) at the Indian sector. One of the CEJ events occurred during quiet time magnetic conditions while the other two were observed during moderately disturbed magnetic conditions. Therefore, the density gradient conditions for the gradient-drift plasmas irregularities development are always established by the presence of sporadic Elayers. Nevertheless, the precise determination of the electric field is still a difficult matter at the E-region heights, despite there be indications that the neutral atmosphere effects are more local than global.

Acknowledgements. C. M. Denardini would like to acknowledge the INPE Research Team on Geomagnetism for providing the geomagnetic data, the ISEA Committee for supporting his attendance to the meeting and the CNPq for the support (305923/20080). H. C. Aveiro (131326/2004-4), Ê. P. A. Olívio (130588/20067), L. C. A. Resende (101536/2006-2) and P. D. S. C. Almeida (105374/2005-9) thank CNPq for theirs fellowship.

Topical Editor K. Kauristie thanks two anonymous referees for their help in evaluating this paper. 


\section{References}

Abdu, M. A., Souza, J. R., Sobral, J. H. A., and Batista, I. S.: Magnetic storm associated disturbance dynamo effects in the low and equatorial latitude ionosphere, in: Recurrent Magnetic Storms: Corotating Solar Wind Streams, edited by: Tsurutani, B. T., McPherron, R., Gonzalez, W. D., Lu, G., Sobral, J. H. A., and Gopalswamy, N., pp. 283-304, American Geophysical Union, Washington, 2006.

Alex, S. and Mukherjee, S.: Local time dependence of the equatorial counter electrojet effect in a narrow longitudinal belt, Earth Planets Space, 53(12), 1151-1161, 2001.

Crochet, M., Hanuise, C. and Broche, P.: HF radar studies of twostream instability during an equatorial counter electrojet, J. Geophys. Res., 84, 5223-5233, 1979.

Denardini, C. M., Abdu, M. A., de Paula, E. R., Wrasse, C. M., and Sobral, J. H. A.: VHF radar observations of the dip equatorial Eregion during sunset in the Brazilian sector, Ann. Geophys., 24, 1617-1623, 2006, http://www.ann-geophys.net/24/1617/2006/.

Denardini, C. M., Abdu, M. A., de Paula, E. R., Sobral, J. H. A., and Wrasse, C. M.: Seasonal characterization of the equatorial electrojet height rise over Brazil as observed by the RESCO $50 \mathrm{MHz}$ back-scatter radar, J. Atmos. Terr. Phys., 67, 1665-1673, 2005.

Farge, M.: Wavelet Transforms and Their Applications to Turbulence, Annu. Rev. Fluid Mech., 24(3), 395-457, 1992.

Faynberg, E. B.: Separation of the geomagnetic field into a normal and an anomalous part, Geomagn. Aeron., 15, 117-121, 1975.

Fejer, B. G. and Kelley, M. C.: Ionospheric Irregularities, Rev. Geophys., 18(2), 401-454, 1980.

Fejer, B. G., Farley, D. T., Balsley, B. B., and Woodman, R. F.: Vertical Structure of VHF Backscattering Region in Equatorial Electrojet and Gradient Drift Instability, J. Geophys. Res., 80(10), 1313-1324, 1975.

Gurubaran, S.: The equatorial counter electrojet: Part of a worldwide current system?, Geophys. Res. Lett., 29(9), 1337, doi:10.1029/2001GL014519, 2002.
Hanuise, C., Mazaudier, C., Vila, P., Blanc, M., and Crochet, M.: Global dynamo simulation of ionospheric currents and their connection with the equatorial electrojet and counter electrojet: A case study, J. Geophys. Res., 88, 253-270, 1983.

Hargreaves, J. K.: The Solar-Terrestrial Environment, 420 pp., Cambridge University Press, Cambridge, 1992.

Heelis, R. A.: Electrodynamics in the low and middle latitude ionosphere: a tutorial, J. Atmos. Terr. Phys., 66, 825-838, 2004.

Kane, R. P.: Geomagnetic field variations, Space Sci. Rev., 18(4), 413-540, 1976.

Mayaud, P. N.: The equatorial counter electrojet-a review of its geomagnetic aspects, J. Atmos. Terr. Phys., 39, 1055-1070, 1977.

Raghavarao, R. and Anandarao, B. G.: Equatorial electrojet and counter electrojet, Indian J. Radio Space Phys., 16, 54-75, 1987.

Reinisch, B. W., Bibl, K., Kitrosser, D. F., Sales, G. S., Tang, J. S., Zhang, Z., Bullett, T. W., and Ralls, J. A.: The Digisonde 256 Ionospheric Sounder, in: World Ionosphere/Thermosphere Study, edited by: Liu, C. H., pp. 350-380, ICSU Scientific Commitee on Solar-Terrestrial Physics (SCOSTEP), Urbana, 1989.

Somayajulu, V. V. and Viswanathan, K. S.: VHF Radar Observations During Equatorial Counter Electrojet Events, Indian J. Radio Space Phys., 16, 380-383, 1987.

Somayajulu, V. V., Selvamurugan, R., Devasia, C. V., and Cherian, L.: VHF Backscatter Radar Observations of Type-I Waves During a Counter Electrojet Event, Geophys. Res. Lett., 21(18), 2047-2050, 1994.

Tarpley, J. D.: Seasonal movement of the Sq current foci and related effects in the equatorial electrojet, J. Atmos. Terr. Phys., 35, 1063-1071, 1973.

Torrence, C. and Compo, G.: A practical guide to wavelet analysis, B.. Am. Meteorol. Soc., 79, 61-78, 1998.

Vertlib, A. B. and Wagner, C. U.: Analysis of geomagnetic Sq variations by the expansion of fields in natural orthogonal components I, Method and problems, Geomagn. Aeron., 10, 509-513, 1970.

Woodman, R. F. and Chau, J. L.: First Jicamarca radar observations of two-stream E region irregularities under daytime counter equatorial electrojet conditions, J. Geophys. Res., 107(A12), A12S1482, doi:10.1029/2002JA009362, 2002. 